\title{
The SPECFIND V2.0 catalogue of radio cross-identifications and spectra
}

\section{SPECFIND meets the Virtual Observatory}

\author{
B. Vollmer, B. Gassmann, S. Derrière, T. Boch, M. Louys, F. Bonnarel, P. Dubois, F. Genova, and F. Ochsenbein
}

CDS, Observatoire astronomique de Strasbourg, UMR 7550, 11 rue de l'université, 67000 Strasbourg, France

e-mail: bvollmer@astro.u-strasbg.fr

Received 13 October 2009 / Accepted 9 December 2009

\section{ABSTRACT}

\begin{abstract}
The new release of the SPECFIND radio cross-identification catalogue, SPECFIND V2.0, is presented. It contains 107488 crossidentified objects with at least three radio sources observed at three independent frequencies. Compared to the previous release the number of entry radio catalogues is increased from 20 to 97 with 115 tables. This large increase was only made possible by the development of four tools at the Centre de Données astronomiques de Strasbourg (CDS) which use the standards and infrastructure of the Virtual Observatory (VO). This was done in the framework of the VO-TECH European Design Study of the Sixth Framework Program. We give an overview of the different classes of radio sources that a user can encounter. Due to the increase of the frequency coverage of the input radio catalogues, this release demonstrates that the SPECFIND algorithm is able to detect spectral breaks around a frequency of $\sim 1 \mathrm{GHz}$.
\end{abstract}

Key words. astronomical data bases: miscellaneous - radio continuum: general

\section{Introduction}

The cross-identification of radio sources observed in the centimeter to meter wavelength domain with different instruments is a rather difficult task because of huge differences in sensitivity, spatial resolution, and the non-simultaneous observations of variable sources. Especially the very different spatial resolutions of single dish telescopes and interferometers are difficult to handle. On the other hand, most sources show a power-law spectral energy distribution in these wavelengths due to synchrotron or thermal emission. Synchrotron emission produces a power law spectrum with a possible cut-off or reversal of the spectral index at low frequencies due to self-absorption or comptonisation. The spectrum of thermal electrons is flat in the optically thin domain.

In Vollmer et al. (2005a) we presented the SPECFIND tool for the extraction of cross-identifications and radio continuum spectra from radio source catalogues contained in the VizieR database of the Centre de Données astronomiques de Strasbourg (CDS). The SPECFIND cross-identification tool takes advantage of the power-law shape of the spectra. In addition, it takes into account the angular resolution of the observations, the source size, and the flux densities observed at a given frequency. The SPECFIND tool also ensures that a radio source cannot be assigned to more than one physical object.

In a first release (SPECFIND V1.0; Vollmer et al. 2005a,b) we cross-identified the sources of the 20 largest radio catalogues in VizieR (Ochsenbein et al. 2000), representing 3.5 million sources. Our work led to more than 700000 independent crossidentifications between sources from different radio catalogues and $\sim 67000$ independent radio spectra with more than two independent frequencies.

The information contained in radio catalogues is heterogeneous and contains different entries (e.g. peak flux or integrated flux) and physical units (e.g. source extent in arcsec, arcmin, or beamsizes). On the other hand, a cross-identification tool needs uniform input: at least a source name, position, and flux density at the measured frequency. The uniformisation of the 20 SPECFIND V1.0 entry catalogues was done by hand. For a significant increase of independent radio cross-identifications, an input of sources from more than a hundred radio catalogues is needed. This goal could only be attained by taking advantage of Virtual Observatory (VO) capabilities, which are described in Sect. 2. The new release of the SPECFIND catalogue is presented in Sect. 3.

\section{Table uniformisation using VO tools}

The Virtual Observatory offers (i) the standards for an efficient table uniformisation; and (ii) the infrastructure to make new tools available to the astronomical community. The aim of this work is two-fold: 1) the discovery of available resources, i.e. radio catalogues, in the VO world; and 2) the extraction and homogenisation of the relevant information from these resources.

Within the framework of the European VO-TECH Design Study we have developed three VO tools at CDS: (i) TABFIND: a tool to search for useful radio catalogues in the Virtual Observatory; (ii) TABUNIF: a tool to extract relevant information from these catalogues and to uniformise the catalogue information; and (iii) CAMEA: a tool to characterise the data, i.e. to include additional metadata necessary for the full usage of the data in the VO, as required in the VO "characterisation" data model (Louys et al. 2008). For example, the angular resolution of the observations is not provided as a standard parameter in the VizieR catalogue description. During the development the tools were kept as general as possible. They can thus be used in other astronomical contexts. We also plan to include 
the extended radio catalogue description gathered by the characterisation tool into VizieR. These tools together with the associated manuals are available at http://eurovotech.org/ twiki/bin/view/VOTech.

\subsection{Registry query tool TABFIND}

This tool identifies VO resources based on unified content descriptors (UCDs). The UCDs are a controlled vocabulary defined by the VO to describe astronomical quantities (Derrière et al. 2004). TABFIND is written in Java and uses XMLDB $\mathrm{API}^{1}$ to get data from the VO registry of resources. The latter is a kind of telephone book where all web servers are listed who comply with VO standards. The user specifies a required set of UCDs. TABFIND searches the VO registry for all catalogues whose descriptions contain these UCDs. For example, in our project the minimum set of parameters needed for the radio cross-identification are source coordinates and a radio flux.

The result of the query is a list of relevant radio catalogues. The catalogues can then be sorted into useful and not useful catalogues by displaying the catalogue descriptions. A workspace permits us to save and restore all actions performed on the catalogues. At the end, a final list of relevant catalogues is established.

\subsection{Data homogenisation tool TABUNIF}

The relevant catalogues obtained from TABFIND can be directly loaded into the data homogenisation tool. TABUNIF creates homogenised data from a heterogeneous set of catalogues. It is written in Java and works on XML tables. In a first step the user specifies a set of columns for the output table which can be based on the list of UCDs ${ }^{2}$. In our case we defined the output columns according to the needs of the SPECFIND crossidentification tool (Vollmer et al. 2005a). In a second step the tool generates an interface where a column of the entry radio catalogue is assigned to a user-specified output column.

The user is free to change the input column that he/she wants to assign to an output column. It is also possible to assign an arithmetic combination of different input columns or conditions on input columns to an output column. As a result the tool generates an ASCII output table for each input radio catalogue. The ASCII output tables can be directly used by the SPECFIND cross-identification tool. Alternatively, the output table can be produced in the VO-compliant VOTable format ${ }^{3}$ for future usage by $\mathrm{VO}$ tools.

\subsection{Characterisation tool CAMEA}

We realised that the description of the radio catalogues in VizieR does not contain all necessary information for the cross-identification. Basic information like the identity of the instrument, frequency, resolution, and observation dates are not included in the catalogue metadata. We therefore decided to develop a third VO tool which permits us to specify this missing information. More generally, it will permit us to create a full description of a VO resource based on the VO characterisation data model (Louys et al. 2008). In the future, CAMEA will help

\footnotetext{
1 http://xmldb-org.sourceforge.net/xapi/

2 http://www.ivoa.net/Documents/latest/UCDlist.html

3 The VOTable format is an XML standard for the interchange of data represented as a set of tables; http://www.ivoa.net/Documents / latest/VOT . html
}

to provide the input for the data homogenisation tool TABUNIF described above. In this way we plan to complement the VizieR metadata of radio catalogues by adding the frequency, angular resolution, observation dates, etc.

\section{SPECFIND V2.0}

The use of the registry query tool and data homogenisation tool enabled us to include 97 radio catalogues and 115 tables (a catalogue corresponds to one reference and can contain multiple tables) from VizieR (Ochsenbein et al. 2000) into the SPECFIND radio cross-identification tool (20 catalogues from Vollmer et al. 2005a (Table 1) and the catalogues listed in Table 2). The number of sources from these catalogues is $3.76 \times 10^{6}$ leading to 107488 cross-identified objects, i.e. objects with at least three flux densities observed at three independent frequencies. Compared to the first release of the SPECFIND V1.0 catalogue this is an increase of available radio sources by $\sim 8 \%$. This relatively small increase is caused by the number of catalogues with a given number of radio sources increasing rapidly while the number of radio sources contained in the catalogue decreases. However, the smaller catalogues often provide the missing third flux density to establish a radio spectrum. For example, in the northern hemisphere there is a multitude of radio objects with available NVSS (1.4 GHz; Condon et al. 1998) and WENSS (325 MHz; Rengelink et al. 1997) flux densities. The surveys at higher frequencies, which were included in SPECFIND V1.0, are rather shallow and thus did not detect the majority of the sources. Observations at high frequencies leading to small catalogues are almost always more sensitive than observations which large catalogues consist of, with the drawback that they are made within small areas on the sky. This is the reason why a modest increase of the sources available for the cross-identification $(\sim 8 \%)$ leads to the significant increase of cross-identified radio objects of $\sim 60 \%$. The source coverage of the first and the second release of the SPECFIND catalogues are shown in Fig. 1.

The SPECFIND V2.0 catalogue is available via Vizier at CDS. It has the same data structure as SPECFIND V1.0. Each radio source represents one line of the catalogue. The radio sources from one physical object are linked via a common sequence number. For each radio source SPECFIND V2.0 gives a flag for extended/confused/complex sources (based on the NVSS), the source name, coordinates, flux density, error of the flux density as used in SPECFIND, number of sources with the same sequence number, slope and abcissa of the radio spectrum, positional difference to the NVSS source which is part of the spectrum, and the difference between the interpolated $20 \mathrm{~cm}$ and the NVSS flux density. In addition, we provide a link to the plot of the radio spectrum and a link to the Aladin applet in which the NVSS/DSS images and the positions of the SPECFIND V2.0 radio sources together with the beam sizes of the different observations are displayed. Moreover, we provide access to the radio sources that are cross-identified only with respect to their position (overlapping beams or extents), but do not fit the radio spectrum.

The distribution of the spectral indices ${ }^{4}$ is shown in Fig. 2. The distribution peaks at $\alpha \sim-0.9$, which is consistent with the result from the first release (see also Zhang et al. 2003). As in SPECFIND V1.0, there is a wing towards positive spectral indices, which is most probably caused by the flattening of the spectrum at low frequencies due to synchrotron self-absorption and at high frequencies due to the emission of thermal electrons.

\footnotetext{
4 The spectral index $\alpha$ is defined by $S_{v} \propto v^{\alpha}$.
} 
Table 1. SPECFIND V1.0 catalogue entries.

\begin{tabular}{|c|c|c|c|c|c|c|c|}
\hline $\begin{array}{l}\text { Catalog } \\
\text { name }\end{array}$ & $\mathrm{I} / \mathrm{S}^{1}$ & $\begin{array}{r}\text { Frequency } \\
(\mathrm{MHz})\end{array}$ & $\begin{array}{r}\text { Resolution } \\
\text { (arcmin) }\end{array}$ & $\begin{array}{r}S_{\min } \\
(\mathrm{mJy})\end{array}$ & $\begin{array}{r}\text { Number of } \\
\text { sources }\end{array}$ & Percentage $^{2}$ & Reference \\
\hline JVAS & I & 8400 & $5.5 \times 10^{-3}$ & 30 & 2246 & 72 & $\begin{array}{l}\text { Patnaik et al. (1992) } \\
\text { Browne et al. (1998) } \\
\text { Wilkinson et al. (1998) }\end{array}$ \\
\hline GB6 & S & 4850 & 3.5 & 18 & 75162 & 59 & Gregory et al. (1996) \\
\hline $87 \mathrm{~GB}$ & S & 4850 & 3.5 & 25 & 54579 & 64 & Gregory \& Condon (1991) \\
\hline BWE & S & 4850 & 3.5 & 25 & 53522 & 61 & Becker et al. (1991) \\
\hline $\mathrm{PMN}$ & S & 4850 & 3.5 & 20 & 50814 & 30 & $\begin{array}{l}\text { Wright et al. }(1994,1996) \\
\text { Griffith et al. }(1994,1995)\end{array}$ \\
\hline MITG & S & 4850 & 2.8 & 40 & 24180 & 52 & $\begin{array}{l}\text { Bennett et al. }(1986) \\
\text { Langston et al. }(1990) \\
\text { Griffith et al. }(1990,1991)\end{array}$ \\
\hline PKS & S & 2700 & 8.0 & 50 & 8264 & 65 & Otrupcek \& Wright (1991) \\
\hline F3R & S & 2700 & 4.3 & 40 & 6495 & 61 & Fürst et al. (1990) \\
\hline FIRST & I & 1400 & 0.083 & 1 & 811117 & 1.5 & White et al. (1998) \\
\hline NVSS & I & 1400 & 0.75 & 2 & 1773484 & 3.6 & Condon et al. (1998) \\
\hline WB & S & 1400 & 10. & 100 & 31524 & 60 & White \& Becker (1992) \\
\hline SUMSS & I & 843 & 0.75 & 8 & 134870 & 1.8 & Mauch et al. (2003) \\
\hline B2 & I & 408 & 8.0 & 250 & 9929 & 72 & $\begin{array}{l}\text { Colla et al. }(1970,1972,1973) \\
\text { Fanti et al. (1974) }\end{array}$ \\
\hline B3 & I & 408 & 5.0 & 100 & 13340 & 66 & Ficarra et al. (1985) \\
\hline MRC & I & 408 & 3.0 & 700 & 12141 & 73 & Douglas et al. (1996) \\
\hline TXS & I & 365 & 0.1 & 250 & 66841 & 57 & Large et al. (1991) \\
\hline WISH & I & 325 & 0.9 & 10 & 90357 & 8.4 & de Breuck et al. (2002) \\
\hline WENSS & I & 325 & 0.9 & 18 & 229420 & 17 & Rengelink et al. (1997) \\
\hline MIYUN & I & 232 & 3.8 & 100 & 34426 & 40 & Zhang et al. (1997) \\
\hline $4 \mathrm{C}$ & I & 178 & 11.5 & 2000 & 4844 & 53 & $\begin{array}{l}\text { Pilkington \& Scott (1965) } \\
\text { Gower et al. (1967) }\end{array}$ \\
\hline $3 \mathrm{CR}$ & I & 178 & 6.0 & 5000 & 327 & 31 & Bennet (1962) \\
\hline $3 \mathrm{C}$ & I & 159 & 10.0 & 7000 & 470 & 3.4 & Edge et al. (1959) \\
\hline
\end{tabular}

Notes. ${ }^{(1)}$ S: single dish; I: interferometer. ${ }^{(2)}$ Percentage of sources with identified spectrum.

The number of objects as a function of the number of catalogued sources contained in an object is shown in Fig. 3. The shape of the distribution is a broken power law with a break at about 12 sources per object. The maximum number of catalogued sources in a physical object is 30 .

The distributions of the spectral indices as a function of the measured or interpolated flux density at $325 \mathrm{MHz}$ from SPECFIND V1.0 and V2.0 are shown in Fig. 4.

In SPECFIND V1.0 the straight, almost horizontal edge of the distribution in the left part of the plot (marked as (a) in the upper panel of Fig. 4) is due to a selection effect. For these low flux density sources with a steep spectrum, SPECFIND found a source at $20 \mathrm{~cm}$ (NVSS) and $50 \mathrm{~cm}$ (WENSS), but none at $6 \mathrm{~cm}$, where the sensitivity of the surveys $(\sim 20 \mathrm{mJy})$ is insufficient. The inclusion of the new radio catalogues at $4850 \mathrm{MHz}$ into SPECFIND V2.0 improved this situation only mildly.

The vertical edge in the lower left part of the plot (marked as (b) in the upper panel of Fig. 4) is mainly due to the limiting flux density of the B3 survey. Here the inclusion of a significant number of new sources at low frequencies $(v<1 \mathrm{GHz}$, mainly the 6C and 7C catalogues) leads to a significant increase of objects with $325 \mathrm{MHz}$ flux densities smaller than $100 \mathrm{mJy}$ and spectral indices smaller than 0 .

\section{Compatibility with SPECFIND V1.0}

The cross-identification of radio sources observed at different frequencies and with considerably different angular resolutions (see Table 2) is a complex task. The details of the SPECFIND cross-identification algorithm are described in Vollmer et al. (2005a). In a first step SPECFIND makes a positional crossidentification accounting for the source extent and resolution of the survey. In a second step the flux densities and associated errors observed at the same frequency are compared and in a third step, a power law is fitted to the flux densities at different frequencies. The cross-identification is done for each catalogued radio source separately, which results in different spectral indices of sources belonging to the same object. In crowded fields with a high source density the cross-identification might not be unique and depends on the weight given to each source in the physical object. The resulting degeneracy in the cross-identification is solved by a self-consistency check of all physical objects found by SPECFIND (for details see Vollmer et al. 2005a). This procedure ensures that a radio source cannot be associated with two different physical objects.

Once sources of new catalogues are added to the input of the SPECFIND cross-identification tool, sources in crowded fields can be redistributed among physical objects, former objects can disappear and new ones can be created. To ensure 
Table 2. Additional SPECFIND V2.0 catalogue entries.

\begin{tabular}{|c|c|c|c|c|c|c|c|}
\hline $\begin{array}{l}\text { Catalog } \\
\text { name }\end{array}$ & $\mathrm{I} / \mathrm{S}^{1}$ & $\begin{array}{r}\text { Frequency } \\
(\mathrm{MHz})\end{array}$ & $\begin{array}{r}\text { Resolution } \\
\text { (arcmin) }\end{array}$ & $\begin{array}{r}S_{\min } \\
(\mathrm{mJy})\end{array}$ & $\begin{array}{r}\text { Number of } \\
\text { sources }\end{array}$ & Percentage $^{2}$ & Reference \\
\hline NEK & I & 31 & 12.0 & 2000 & 703 & 4 & Kassim (1988) \\
\hline $8 \mathrm{C}$ & I & 38 & 4.5 & 700 & 5859 & 66 & Hales et al. (1995) \\
\hline CRJ2004 & I & 74 & 0.42 & 150 & 949 & 63 & Cohen et al. (2004) \\
\hline VLSS & I & 74 & 1.33 & 400 & 68308 & 66 & Cohen et al. (2007) \\
\hline TRC2006b & I & 74 & 0.5 & 0.3 & 725 & 0.2 & Tasse et al. (2006) \\
\hline Cula & I & 80 & 3.7 & 2000 & 2173 & 49 & Slee (1995) \\
\hline $6 \mathrm{C}$ & I & 151 & 4.2 & 300 & 27666 & 84 & $\begin{array}{l}\text { Baldwin et al. (1985) } \\
\text { Hales et al. }(1988 / 80 / 91 / 93)\end{array}$ \\
\hline $7 \mathrm{C}$ & I & 151 & 1.17 & 200 & 43683 & 77 & Hales et al. (2007) \\
\hline Culb & I & 160 & 1.85 & 1000 & 2042 & 66 & Slee (1995) \\
\hline TRB2007a & I & 240 & 0.25 & 6 & 466 & 63 & Tasse et al. (2007) \\
\hline TRC2006a & I & 325 & 0.12 & 2.5 & 843 & 47 & Tasse et al. (2006) \\
\hline WSTB90 & I & 327 & 1.5 & 3 & 407 & & Oort et al. (1988) \\
\hline W93a & I & 327 & 1.0 & 1 & 4157 & 32 & Wieringa (1993) \\
\hline WSTB2 & I & 327 & 1.5 & 10 & 309 & & Righetti et al. (1988) \\
\hline WSRTGP & I & 327 & 1.0 & 5 & 3984 & 14 & Taylor et al. (1996) \\
\hline PSRa & & 400 & 1.0 & 0.1 & 561 & 0.4 & Taylor et al. (1993) \\
\hline $32 \mathrm{P}$ & I & 408 & 4.0 & 30 & 494 & 61 & Leahy \& Roger (1996) \\
\hline $5 \mathrm{C} 5$ & I & 408 & 1.5 & 10 & 214 & 60 & Pearson (1975) \\
\hline $5 \mathrm{C} 6$ & I & 408 & 1.5 & 6 & 267 & 68 & Pearson (1978) \\
\hline $5 \mathrm{C} 7$ & I & 408 & 1.5 & 10 & 235 & 41 & Pearson (1978) \\
\hline $5 \mathrm{C} 12$ & I & 408 & 1.5 & 2 & 308 & 70 & Benn et al. (1982) \\
\hline $5 \mathrm{C} 13$ & I & 408 & 1.5 & 12 & 238 & 60 & Benn (1995) \\
\hline $51 \mathrm{P}$ & I & 408 & 4.0 & 80 & 383 & 31 & Green \& Riley (1995) \\
\hline $5 \mathrm{C} 12 \mathrm{a}$ & I & 408 & 1.33 & 5 & 680 & 49 & Benn \& Kenderdine (1991) \\
\hline DRAOP & I & 408 & 3.5 & & 915 & 62 & $\begin{array}{l}\text { Landecker \& Caswell (1983) } \\
\text { VizieR VIII } / 55^{3}\end{array}$ \\
\hline CGPSEa & I & 408 & 3.0 & 10 & 140 & 24 & Kerton et al. (2007) \\
\hline $\mathrm{PSRb}$ & & 600 & 1.0 & 0.4 & 352 & 0.4 & Taylor et al. (1993) \\
\hline W93b & I & 608 & 0.5 & 1 & 1693 & 62 & Wieringa (1993) \\
\hline TRB2007b & I & 610 & 0.11 & 1 & 1037 & 35 & Tasse et al. (2007) \\
\hline FLSGMRT & I & 610 & 0.1 & 0.1 & 3944 & 1 & Garn et al. (2007) \\
\hline NAIC & S & 611 & 12.6 & 350 & 3122 & 45 & Durdin et al. (1975) \\
\hline MOST & I & 843 & 0.733 & 40 & 348 & 59 & Jones \& McAdam (1992) \\
\hline MGPS2 & I & 843 & 0.75 & 10 & 48850 & 3 & Murphy et al. (2007) \\
\hline ATESP & I & 1400 & 0.23 & 0.3 & 3370 & 1 & Wieringa \& Ekers (2000) \\
\hline PSRc & & 1400 & 1.0 & 0.1 & 445 & 0.4 & Taylor et al. (1993) \\
\hline OWH82 & S & 1400 & 10.0 & 100 & 487 & 63 & Owen et al. (1982) \\
\hline GPSR & I & 1400 & 0.083 & 5 & 1992 & 11 & Zoonematkermani et al. (1990) \\
\hline LO95 & I & 1400 & 0.25 & 10 & 375 & & Ledlow \& Owen (1995) \\
\hline PDF & I & 1400 & 0.15 & 0.1 & 1079 & 0.1 & Hopkins et al. (1998) \\
\hline VIRMOS & I & 1400 & 0.1 & 0.1 & 1103 & 4 & Bondi et al. (2003) \\
\hline FHW95a & S & 1400 & 15.2 & 40 & 192 & 45 & Filipovic et al. (1995) \\
\hline WST32 & I & 1400 & 1.2 & 10 & 215 & 29 & Fanti et al. (1981) \\
\hline $37 \mathrm{~W}$ & I & 1400 & 0.6 & 1 & 53 & 28 & Walterbos et al. (1985) \\
\hline ELAISR & I & 1400 & 0.25 & 0.1 & 965 & 1 & Ciliegi et al. (1999) \\
\hline MGC2004 & I & 1400 & 0.23 & 0.03 & 1048 & 1 & Morganti et al. (2004) \\
\hline WBH2005 & I & 1400 & 0.1 & 2 & 6919 & 4 & White et al. (2005) \\
\hline RRF1 & S & 1410 & 9.4 & 100 & 884 & & Reich et al. (1990) \\
\hline RRF & S & 1410 & 9.4 & 80 & 1830 & & Reich et al. (1997) \\
\hline QC93 & $S$ & 1410 & 10.0 & 400 & 171 & 57 & Quiniento \& Cersosimo (1993) \\
\hline $\mathrm{CCH} 85$ & I & 1411 & 0.33 & 5 & 208 & 9 & Coleman et al. (1985) \\
\hline WSTB & I & 1412 & 0.385 & 1 & 536 & & Windhorst et al. (1984) \\
\hline WSTB1 & I & 1412 & 0.385 & 1 & 359 & & Oort (1987) \\
\hline $33 \mathrm{P}$ & $\mathrm{I}$ & 1420 & 1.0 & 3 & 255 & 36 & Leahy \& Roger (1996) \\
\hline
\end{tabular}

Notes. ${ }^{(1)}$ S: single dish; I: interferometer. ${ }^{(2)}$ Percentage of sources with identified spectrum. Only unambiguous source names could be counted. (3) This catalogue is a compilation of tables in 27 articles. Landecker \& Caswell (1983) is the first reference. 
Table 2. continued.

\begin{tabular}{|c|c|c|c|c|c|c|c|}
\hline $\begin{array}{l}\text { Catalog } \\
\text { name }\end{array}$ & $\mathrm{I} / \mathrm{S}^{1}$ & $\begin{array}{r}\text { Frequency } \\
(\mathrm{MHz})\end{array}$ & $\begin{array}{r}\text { Resolution } \\
(\text { arcmin) }\end{array}$ & $\begin{array}{r}S_{\min } \\
(\mathrm{mJy})\end{array}$ & $\begin{array}{r}\text { Number of } \\
\text { sources }\end{array}$ & percentage $^{2}$ & Reference \\
\hline FBR2002 & I & 1420 & 1.633 & 3 & 534 & 43 & Filipovic et al. (2002) \\
\hline CGPSEb & I & 1420 & 1.0 & 3 & 140 & 27 & Kerton et al. (2007) \\
\hline RLM94 & I & 1465 & 0.075 & 5 & 725 & 42 & Roettgering et al. (1994) \\
\hline P82_1 & I & 1465 & 0.43 & 200 & 404 & 71 & Perley (1982) \\
\hline NEP & I & 1490 & 0.333 & 1 & 2435 & 16 & Kollgaard et al. (1994) \\
\hline DC78 & S & 2370 & 2.7 & 1 & 858 & 29 & Dressel \& Condon (1978) \\
\hline FBR2002a & I & 2370 & 0.667 & 1 & 697 & 33 & Filipovic et al. (2002) \\
\hline FHW95b & S & 2450 & 8.85 & 30 & 334 & 54 & Filipovic et al. (1995) \\
\hline FORa & S & 2695 & 4.78 & 20 & 221 & 81 & Forkert \& Altschuler (1987) \\
\hline RFS & S & 2695 & 4.9 & 30 & 1212 & 27 & Reich et al. (1984) \\
\hline RGB11 & $S$ & 2700 & 4.3 & 50 & 697 & 50 & Reich et al. (2000) \\
\hline PBD2003 & S & 2700 & 8.0 & 100 & 1432 & 9 & Paladini et al. (2003) \\
\hline KMP90 & S & 4730 & 2.8 & 15 & 752 & 85 & Kulkarni et al. (1990) \\
\hline $\mathrm{FORb}$ & S & 4750 & 2.71 & 25 & 227 & 85 & Forkert \& Altschuler (1987) \\
\hline FHW95c & S & 4750 & 4.8 & 15 & 368 & 53 & Filipovic et al. (1995) \\
\hline NAICGB & S & 4775 & 2.8 & 8 & 2453 & 65 & Lawrence et al. (1983) \\
\hline A86 & S & 4760 & 2.8 & 15 & 882 & 74 & Altschuler (1986) \\
\hline FBR2002b & I & 4800 & 0.5 & 1 & 75 & 40 & Filipovic et al. (2002) \\
\hline GDP & I & 4850 & 0.5 & 0.3 & 253 & 8 & Gregorini et al. (1994) \\
\hline CAB95 & S & 4850 & 3.5 & 25 & 351 & 76 & Condon et al. (1995) \\
\hline JRB99 & I & 4860 & 0.03 & 1 & 298 & 28 & Jackson et al. (1999) \\
\hline FPD2001a & I & 4860 & $6.7 e-3$ & 1 & 213 & 36 & Fanti et al. (2001) \\
\hline ADP79 & S & 4875 & 2.6 & 100 & 569 & 19 & Altenhoff et al. (1979) \\
\hline RGB & I & 4885 & 0.067 & 13 & 1861 & 56 & Laurent-Muehleisen et al. (1997) \\
\hline P82_2 & I & 4885 & 0.1 & 200 & 404 & 82 & Perley (1982) \\
\hline $\mathrm{KR}$ & I & 4890 & 0.07 & 10 & 195 & 41 & Fich (1986) \\
\hline GPSR5 & I & 4900 & 0.067 & 1 & 1286 & 3 & Becker et al. (1994) \\
\hline Slee & I & 4900 & 0.67 & 0.2 & 177 & 21 & Slee et al. (1998) \\
\hline HCS79 & & 5000 & 0.54 & 240 & 702 & 3 & Haynes et al. (1979) \\
\hline RGB6 & S & 5000 & 2.4 & 40 & 729 & 56 & Reich et al. (2000) \\
\hline GPA2 & S & 8350 & 9.7 & 900 & 555 & 6 & Langston et al. (2000) \\
\hline CLASS & I & 8400 & $5.5 \mathrm{e}-3$ & 0.1 & 21486 & 39 & Myers et al. (2003) \\
\hline FPD2001b & I & 8460 & $3.3 e-3$ & 0.2 & 199 & 33 & Fanti et al. (2001) \\
\hline FHW95d & S & 8550 & 2.7 & 20 & 205 & 47 & Filipovic et al. (1995) \\
\hline FBR2002c & I & 8640 & 0.27 & 1 & 54 & 41 & Filipovic et al. (2002) \\
\hline NKB95 & S & 10550 & 1.15 & 3 & 202 & 57 & Niklas et al. (1995) \\
\hline B3VLA & $S$ & 10600 & 1.15 & 10 & 981 & 80 & Gregorini et al. (1998) \\
\hline RGB2 & S & 10700 & 1.2 & 30 & 698 & 53 & Reich et al. (2000) \\
\hline GPA1 & S & 14350 & 6.6 & 2000 & 365 & 7 & Langston et al. (2000) \\
\hline $9 \mathrm{C}$ & I & 15000 & 0.42 & 25 & 242 & 71 & Waldram et al. (2003) \\
\hline
\end{tabular}

Notes. ${ }^{(1)}$ S: single dish; I: interferometer. ${ }^{(2)}$ Percentage of sources with identified spectrum. Only unambiguous source names could be counted.

the compatibility between the SPECFIND V1.0 and V2.0 catalogues, we developed a tool to check the coherence between these catalogues. This tool searches for (i) radio sources of SPECFIND V1.0 which are not found in V2.0; and (ii) radio sources of a physical object in SPECFIND V1.0 which are found in different physical objects in V2.0. The tool displays the V1.0 and V2.0 object lists together with the V1.0 and V2.0 spectra. As an additional step the user can display the NVSS $20 \mathrm{~cm}$ image within Aladin (Bonnarel et al. 2000) together with the source positions and the beam sizes (angular resolution) (Figs. 5-9). This information allowed us to either (i) merge both spectra or to keep the (ii) the V1.0; or (iii) V2.0 spectrum. In this way we visualised and modified about 1000 physical objects in the SPECFIND V2.0 catalogue.
This procedure also allowed us to detect complicated cases of the radio cross-identification. Below we present the five major classes of physical objects that a user finds in the SPECFIND V2.0 catalogue: (i) well-behaved; (ii) extended; (iii) complex; (iv) physical double; and (v) unphysical double sources. Sources of class (ii)-(v) can be recognised by a spread of the spectral indices $\alpha$ of the sources contained in a physical object which is larger than the uncertainty due to the flux density errors ( $\Delta \alpha>0.3$; Vollmer et al. 2005b). We decided to leave all these sources in the catalogue. We therefore caution the user against a blind use of the SPECFIND V2.0 catalogue. To help the user, we provide flags for sources which (i) have at least one neighbouring NVSS source within a radius of $2^{\prime}$ (possible confusion); (ii) have deconvolved sizes larger than $45^{\prime \prime}$ in the NVSS 


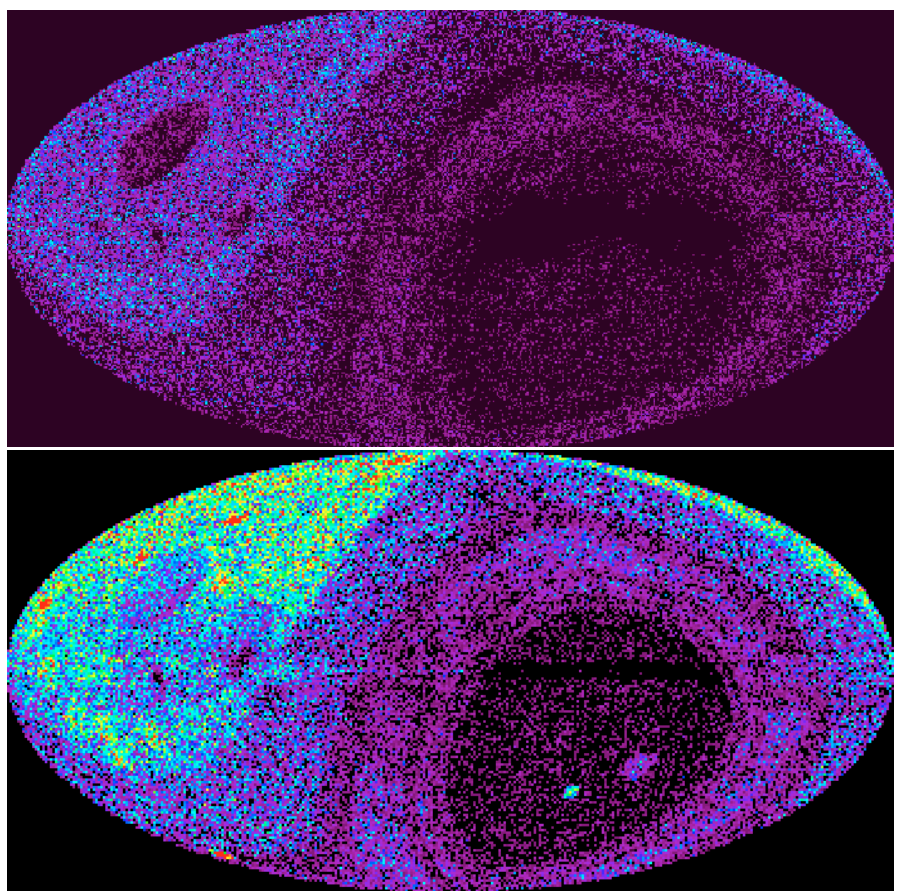

Fig. 1. Sky coverage of radio sources. In both images black/blue/red corresponds to 0/27/64 objects per pixel. Upper panel: from the first release; lower panel: from the SPECFIND V2.0 catalogue.

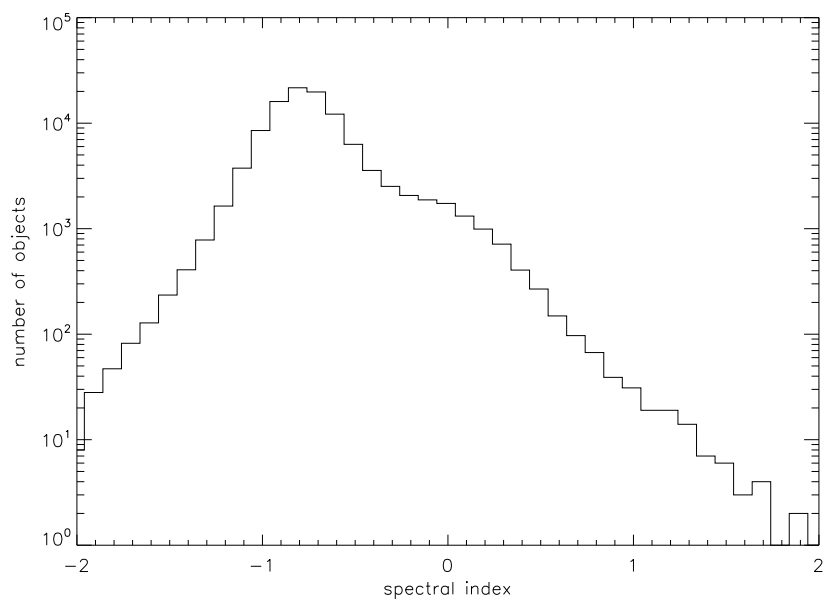

Fig. 2. Number distribution of spectral indices.

catalogue (extended sources), and (iii) are marked as complex in the NVSS catalogue.

\subsection{Well-behaved sources}

The vast majority of the SPECFIND V2.0 objects are wellbehaved (Fig. 5), i.e. they are unresolved or marginally resolved in most of the surveys and exhibit consistent spectral indices for all sources (the uncertainty of the spectral index is \pm 0.3 ; Vollmer et al. 2005a). In the case of sources which are only present in one of the catalogues SPECFIND V1.0 or V2.0, we merged the spectra of all these well-behaved sources.

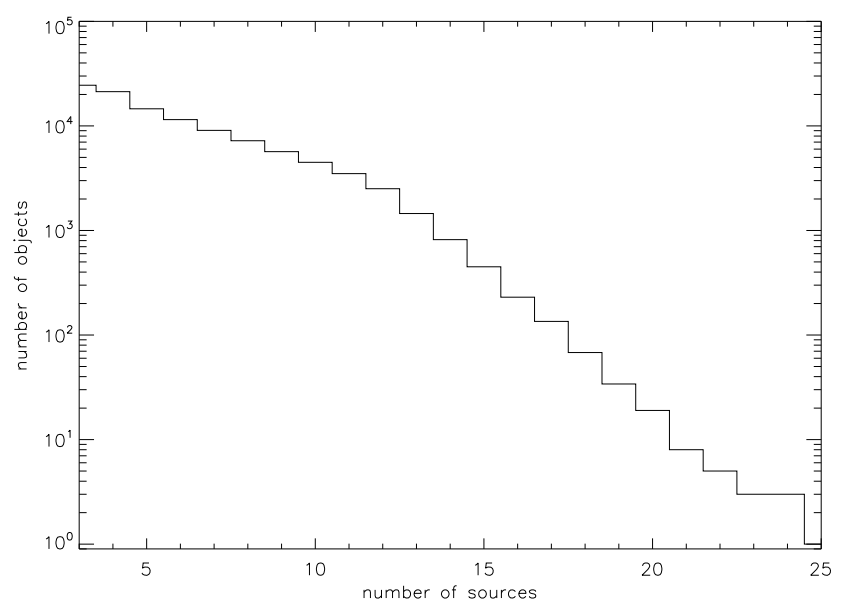

Fig. 3. The number of sources as a function of the number of independent points in the radio spectrum.
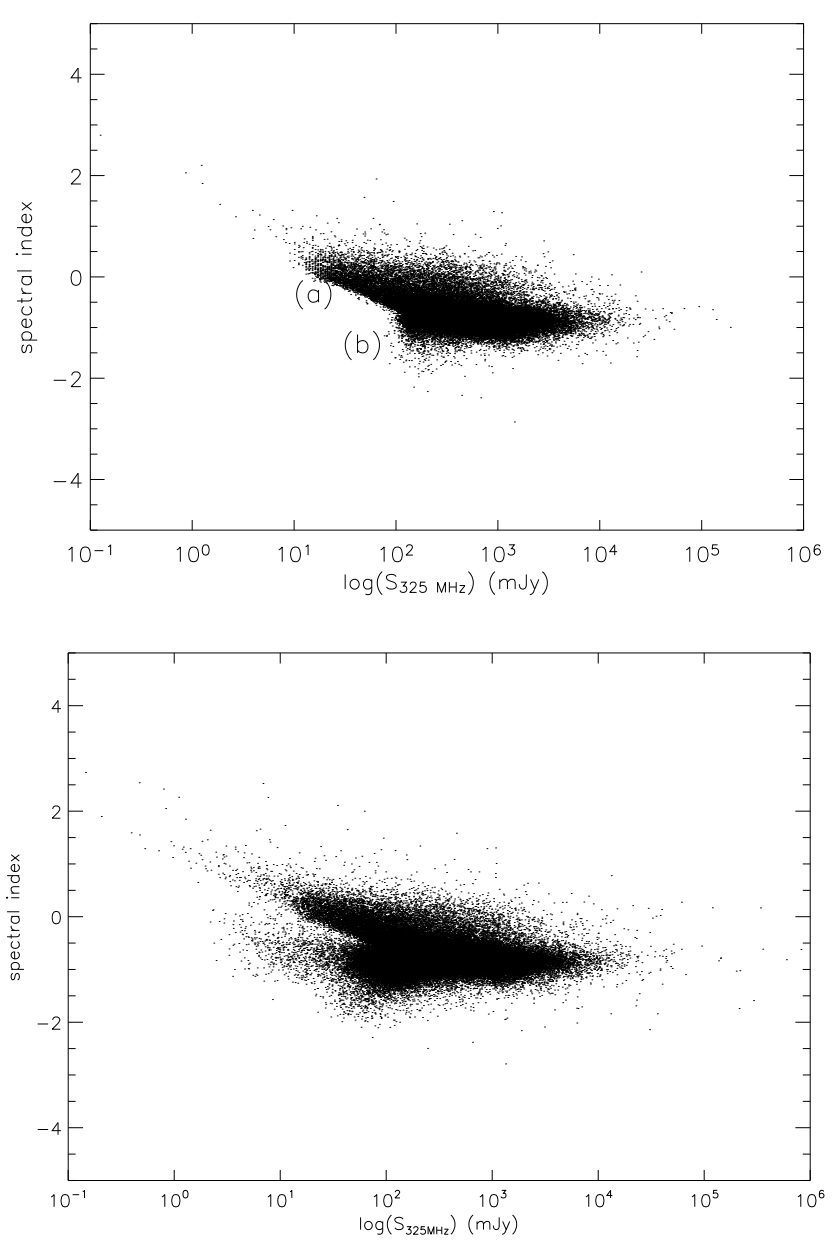

Fig. 4. Spectral index as a function of $325 \mathrm{MHz}$ flux density. Upper panel: SPECFIND V1.0 (Vollmer et al. 2005a). Lower panel: SPECFIND V2.0.

\subsection{Extended sources}

The second class of sources are those which are extended with respect to the mean resolution of the input catalogues, which is $\sim 1-2^{\prime}$. An example for such a source is shown in Fig. 6. At low frequencies these objects have radio fluxes from low resolution 


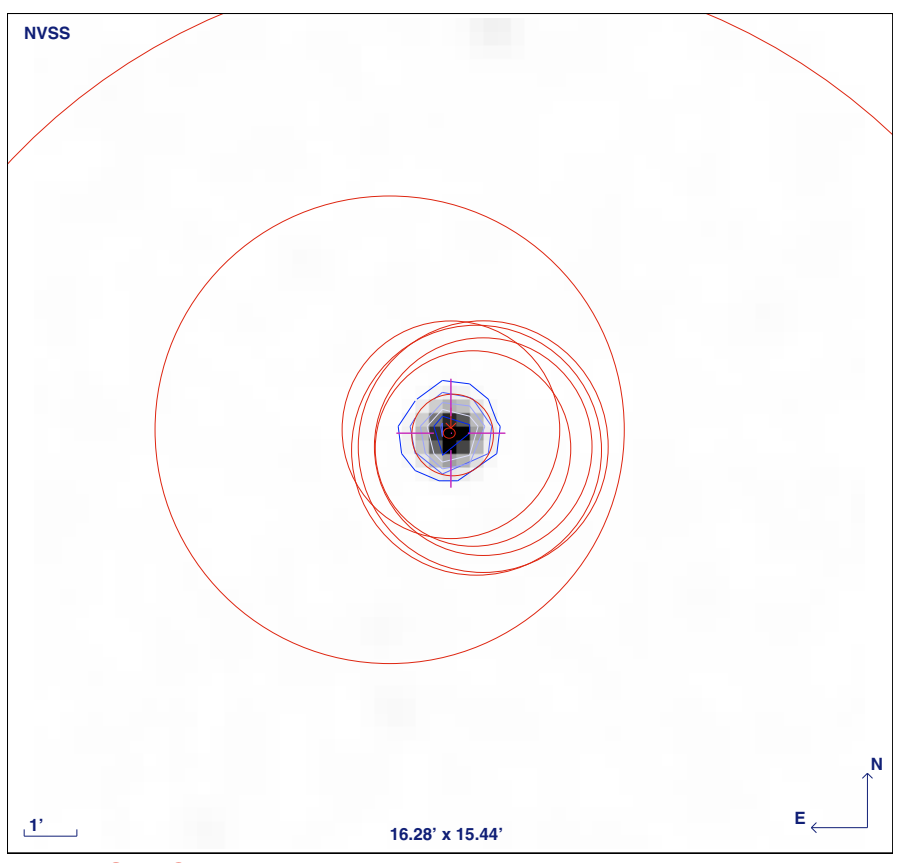

SPECFIND spectrum \#107144

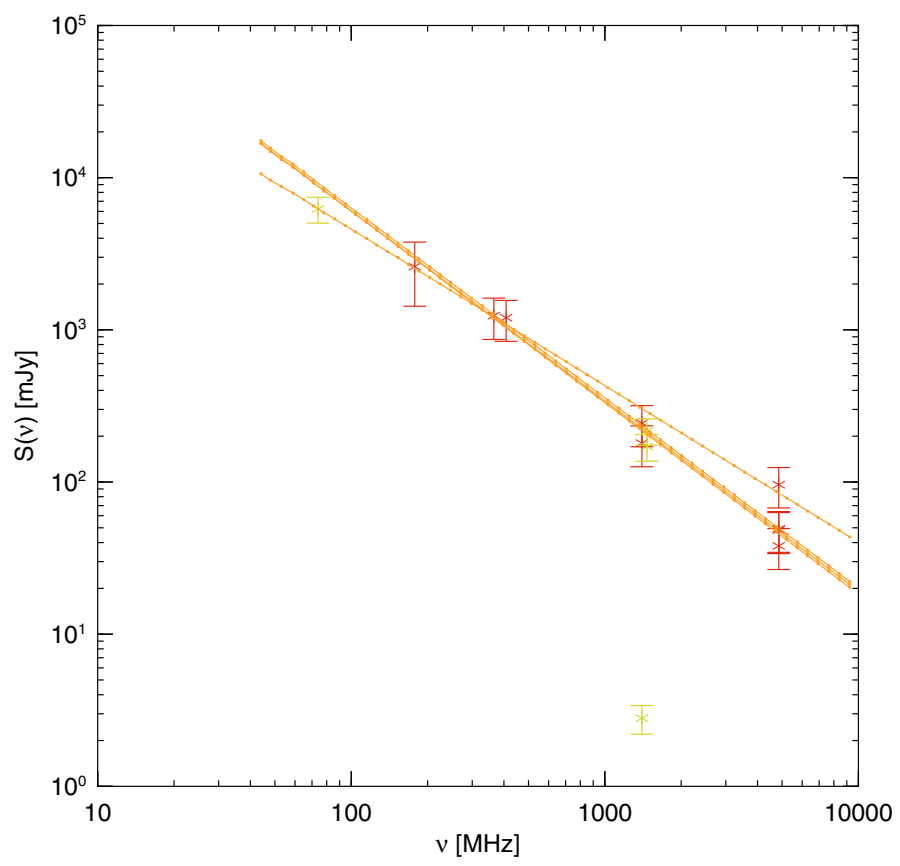

Fig. 5. Well-behaved sources - TXS 2112+158. Upper panel: Aladin view of the NVSS image, the positions of the radio sources and the beamsizes of the radio surveys. Lower panel: Vizier view of the radio spectrum. Red symbols: Specfind V2.0; green symbols: waste, i.e. source with overlapping beams that do not fit the radio spectrum.

surveys in which the source is unresolved. However, at high frequencies the radio fluxes are provided by high resolution surveys. The source is thus resolved leading to a flux density which is smaller than the total flux density. The spectrum of the physical object has two different slopes: one which is fitted to the flux density of the unresolved sources and one which is fitted to the flux density of the resolved sources. In these cases the user has to verify the resolutions of the data points and to make a choice to which points he or she wants to fit a power law. Extended sources thus exhibit different spectral indices $(\Delta \alpha>0.3)$ within the same

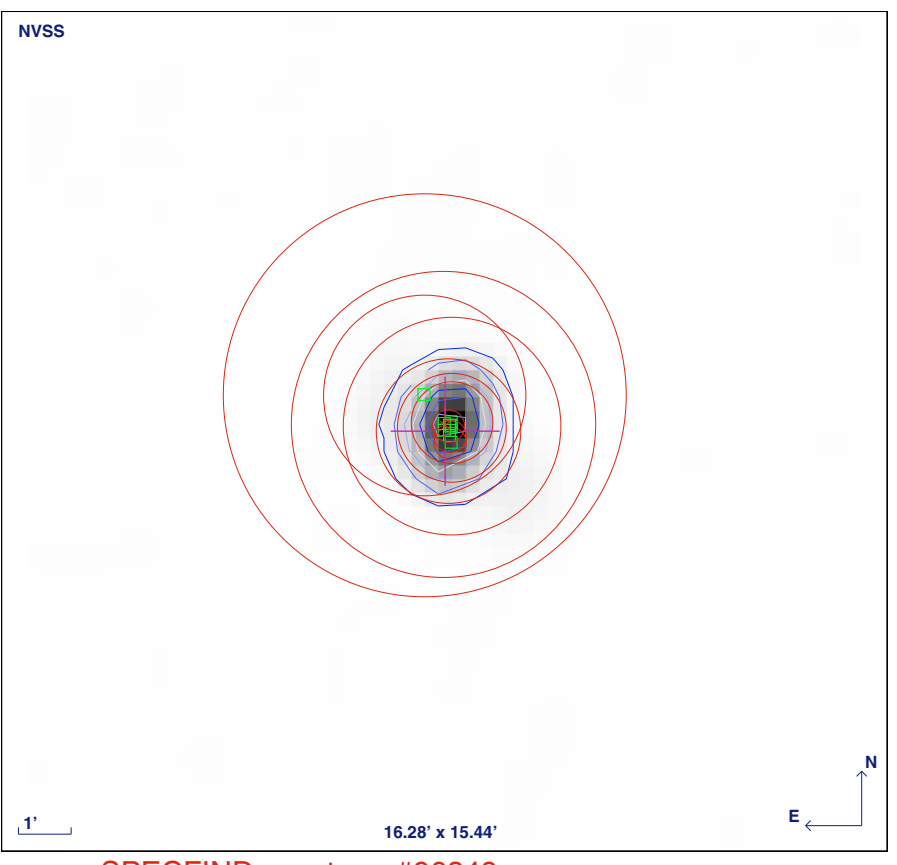

SPECFIND spectrum \#96349

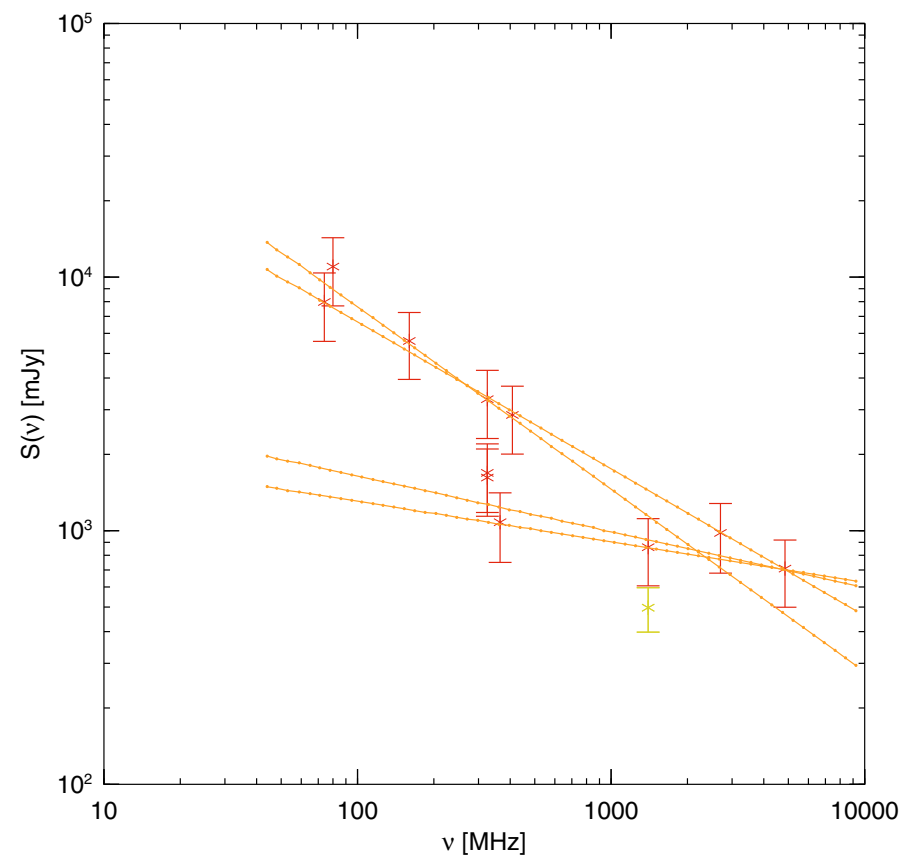

Fig. 6. Extended sources - TXS 2300-189. Upper panel: Aladin view of the NVSS image, the positions of the radio sources and the beamsizes of the radio surveys. Lower panel: Vizier view of the radio spectrum. Red symbols: Specfind V2.0; green symbols: waste, i.e. source with overlapping beams that do not fit the radio spectrum.

frequency range (between 100 and $500 \mathrm{MHz}$ in the example of Fig. 6).

\subsection{Complex sources}

Nearby large Galactic radio sources often display a complex structure (Fig. 7). In the presence of a sufficient number of observations at different frequencies the SPECFIND algorithm identifies power laws, but as for the extended sources, there are multiple spectral indices within the same frequency range (here between $100 \mathrm{MHz}$ and $10 \mathrm{GHz}$ ). In the case of such complex 


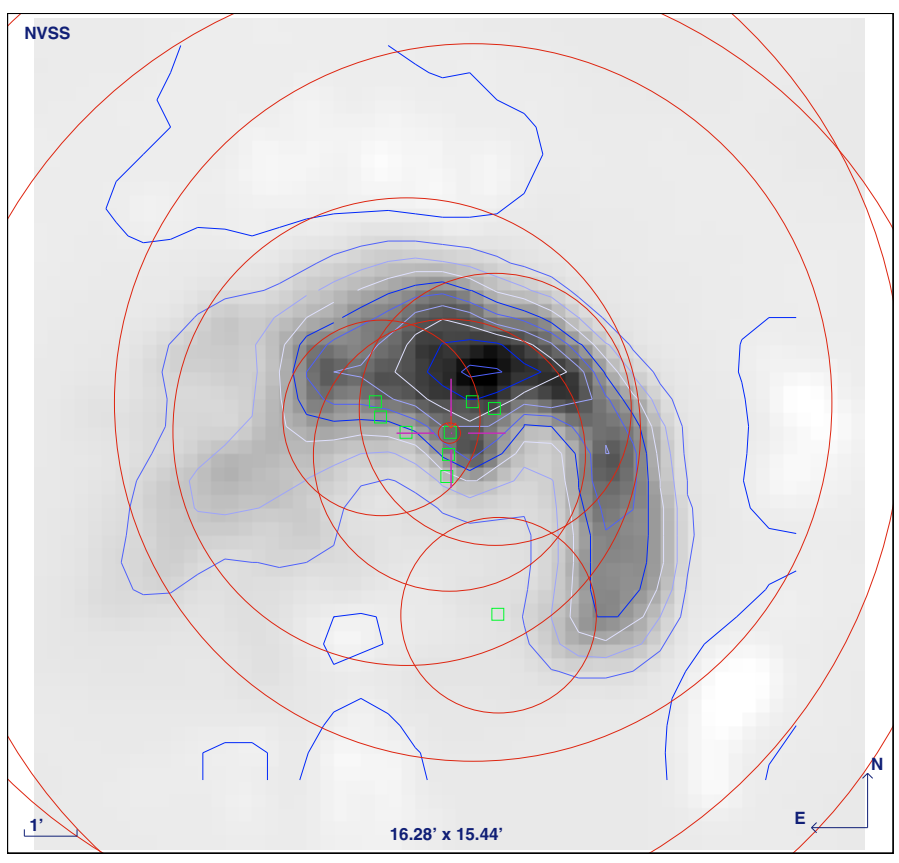

SPECFIND spectrum \#86556

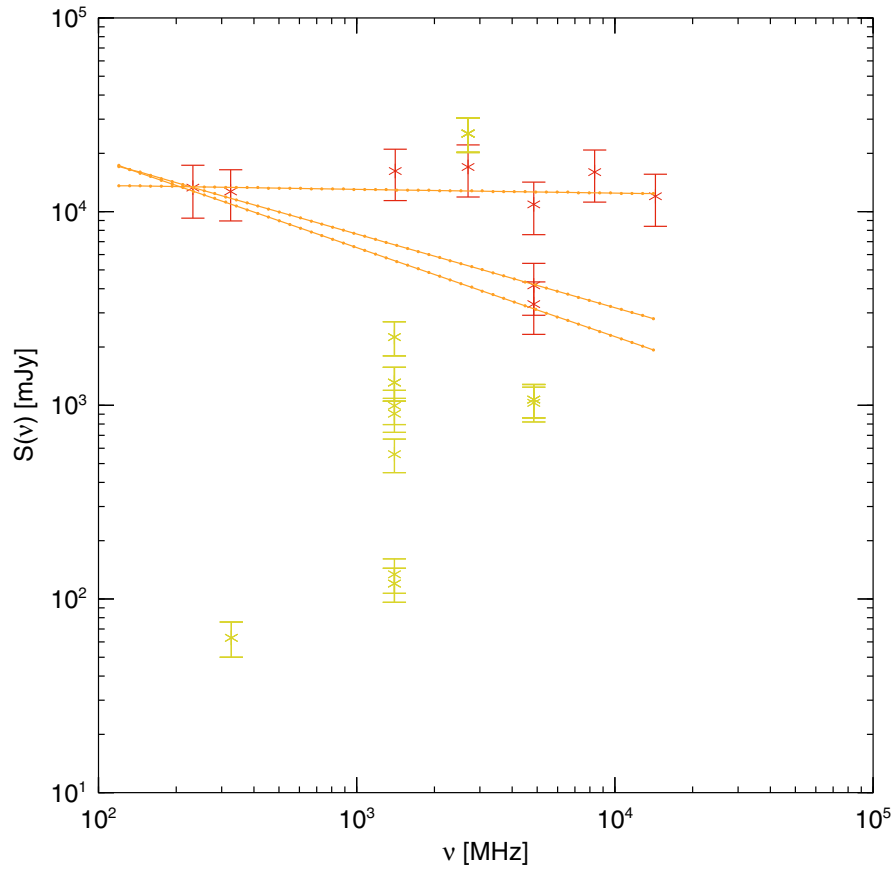

Fig. 7. Complex sources - WN B2040.8+4246. Upper panel: Aladin view of the NVSS image, the positions of the radio sources and the beamsizes of the radio surveys. Lower panel: vizier view of the radio spectrum. Red symbols: Specfind V2.0; green symbols: waste, i.e. source with overlapping beams that do not fit the radio spectrum.

sources the user has to carefully inspect all resolutions and, if necessary, all source extents in the original catalogues in VizieR.

\subsection{Physical double sources}

Since the typical resolution of the SPECFIND entry surveys is $\sim 2^{\prime}$ (Table 2), radio sources which are separated by less than this distance will most frequently end up in one object in the SPECFIND catalogue (Fig. 8). There are observations with large

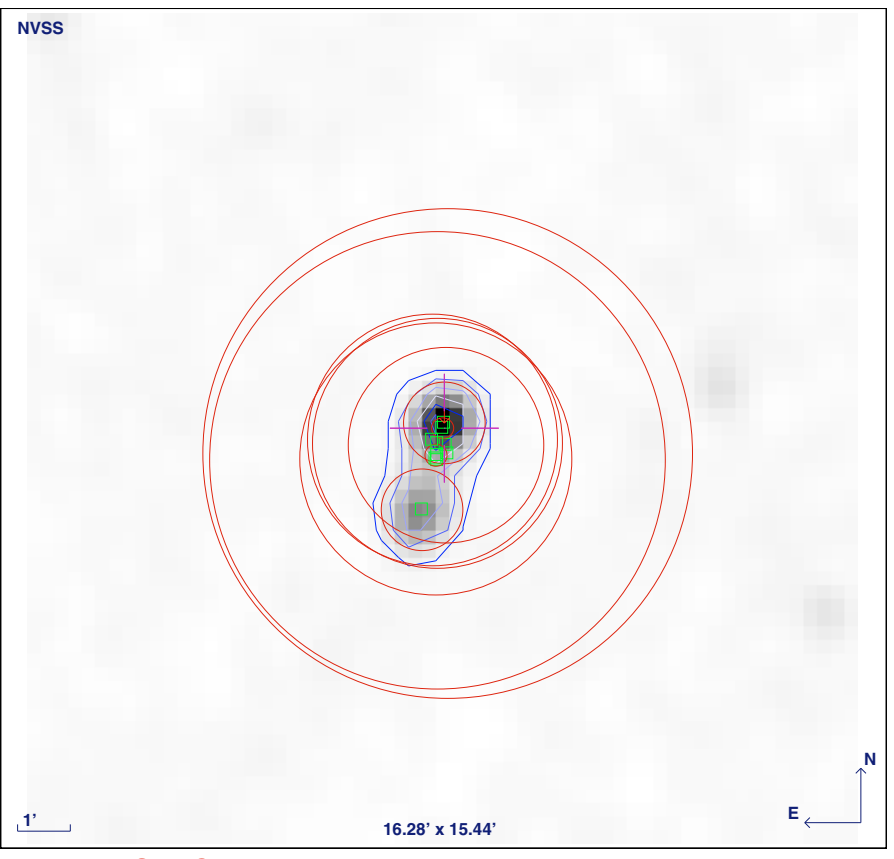

SPECFIND spectrum \#81070

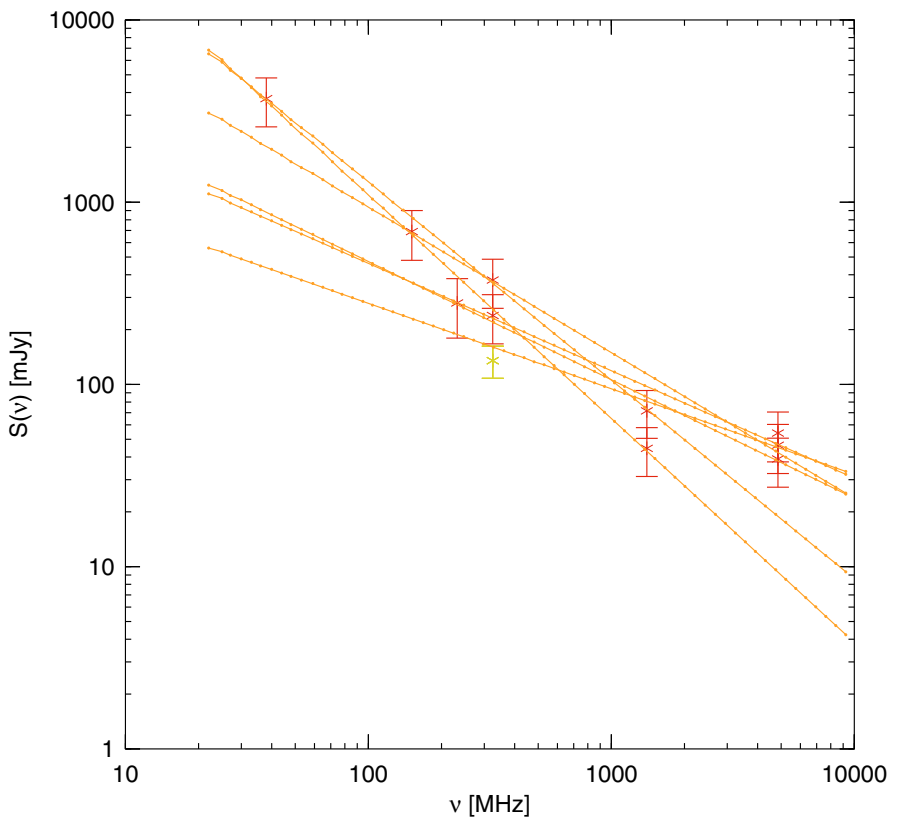

Fig. 8. Possibly physical double sources - WN B1853.1+6226A. Upper panel: Aladin view of the NVSS image, the positions of the radio sources and the beamsizes of the radio surveys. Lower panel: Vizier view of the radio spectrum. Red symbols: Specfind V2.0; green symbols: waste, i.e. source with overlapping beams that do not fit the radio spectrum. beamwidths which comprise the two sources and observations with higher resolution which resolve the two sources. In a physical double source, i.e. the radio lobes of an active galactic nucleus, the two sources often differ in flux density, size, and spectrum. Many double lobe radio sources show an intrinsic asymmetry in their lobes, probably due to Doppler-boosting and jet inclination. The different resolutions of the surveys and the detection of different source components leads to a dispersion of the spectral indices in the composite spectrum. 


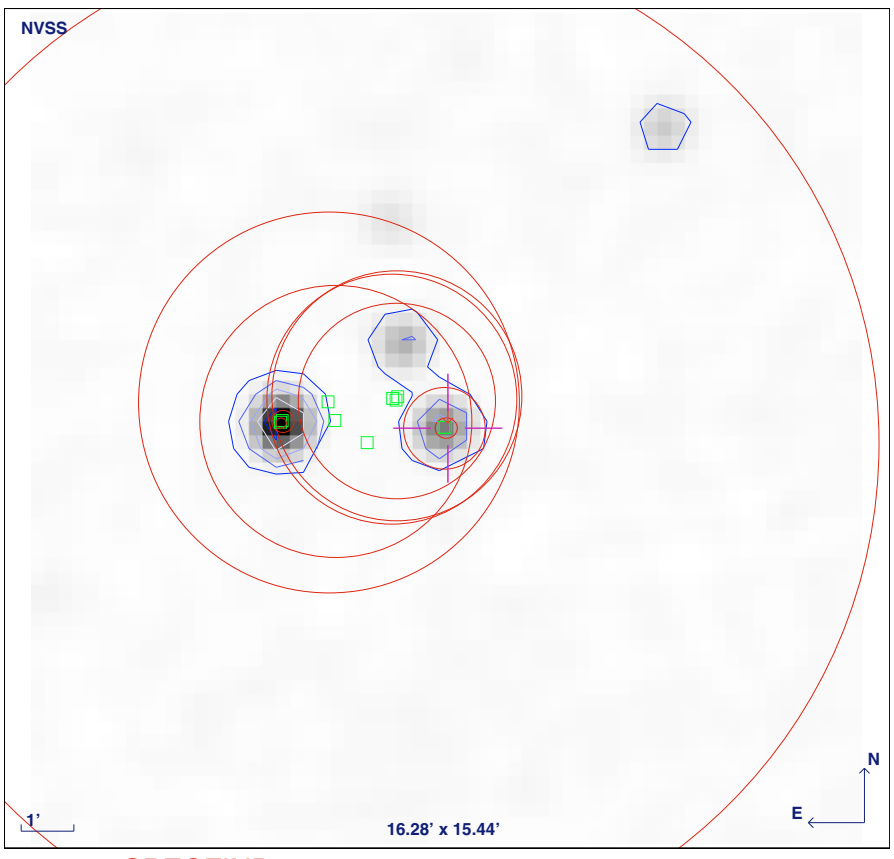

SPECFIND spectrum \#92639

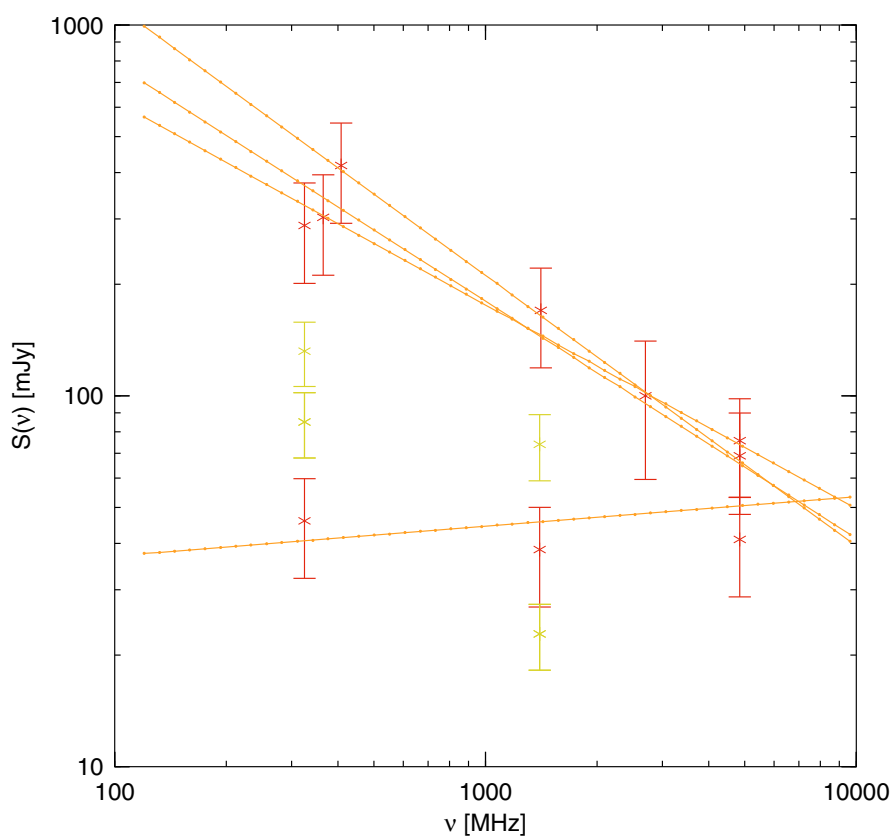

Fig. 9. Unphysical double sources - WN B2228.2+5940A. Upper panel: Aladin view of the NVSS image, the positions of the radio sources and the beamsizes of the radio surveys. Lower panel: Vizier view of the radio spectrum. Red symbols: Specfind V2.0; green symbols: waste, i.e. source with overlapping beams that do not fit the radio spectrum.

\subsection{Unphysical multiple sources}

Other sources which are separated by less than $2^{\prime}$ are not physically related, i.e. the sources are only close in projection (Fig. 9). When these sources exhibit two largely different spectral indices, they can be separated easily with the help of the radio spectrum. If these sources have about the same spectral index, the user has to rely on the NVSS image.

\section{Spectral breaks}

As described in Vollmer et al. (2005a) the SPECFIND algorithm is able to detect a spectral break if enough data points at independent frequencies are available. Due to the increased number of input frequencies in the SPECFIND V2.0 catalogue, we could identify 18 sources which show a spectral break (Fig. 10). Since the frequency coverage of the input survey ranges between $\sim 100 \mathrm{MHz}$ and $\sim 10 \mathrm{GHz}$, the break is located in the middle of this interval around $1 \mathrm{GHz}$. These giga Hertz peaked sources (GPS) are powerful $\left(\log P_{1.4 \mathrm{GHz}}>25 \mathrm{~W} \mathrm{~Hz}^{-1}\right)$ and compact $(<1 \mathrm{kpc})$ extragalactic radio sources, which show a convex radio spectrum peaking between $500 \mathrm{MHz}$ and $10 \mathrm{GHz}$ in the observer's frame (for a review see O'Dea 1998). The physical mechanism responsible for the turnover of the spectrum is still unclear and two competing models are proposed: the synchrotron self-absorption caused by dense plasma within the source or the free-free absorption caused by a screen external to the source. GPS sources are associated with either quasars or galaxies. All but one object of Fig. 10 were already included in the SPECFIND V1.0 catalogue, but the limited frequency range often prevented the detection of the spectral break. Four objects are included in the GPS sample of Vollmer et al. (2008) which was based on SPECFIND V1.0 objects showing an inverted radio spectrum.

\section{Accuracy of radio catalogues}

The cross-identification realised for SPECFIND V2.0 allowed us to evaluate the accuracy in position and flux density of the 97 radio catalogues with respect to the NVSS source catalogue. We do not find significant systematic offset in position or flux densities between the entry catalogues (Table 2) and the NVSS catalogue.

\section{Summary}

We present the second release of the SPECFIND catalogue, SPECFIND V2.0, which is available at CDS's VizieR ${ }^{5}$. The catalogue contains 107488 cross-identified objects with at least three radio sources observed at three independent frequencies. The cross-identification algorithm is based on proximity, source extent, survey resolution, flux density at the same frequency, and power law spectra at different frequencies (Vollmer et al. 2005a). We increased the number of entry catalogues from 20 to 97 with 115 tables (Table 2). This large increase was only made possible by the development of four tools at CDS which use the standards and infrastructure of the Virtual Observatory (VO). This was done in the framework of the VO-TECH Design Study. The first three tools described below are not specificly designed for radio data and can be used for other purposes. Together with the associated manuals they are available at http: //eurovotech. org/twiki/bin/view/VOTech. These tools are:

1. TABFIND: discovery of relevant resources based on unified content descriptors (see Sect. 2.1).

2. TABUNIF: Uniformisation of a heterogeneous set of catalogues (see Sect. 2.2).

3. CAMEA: Description of the catalogue data (see Sect. 2.3).

4. A SPECFIND-specific tool to insure the compatibility between two successive releases of the SPECFIND catalogue.

5 http://vizier.u-strasbg.fr/viz-bin/VizieR 


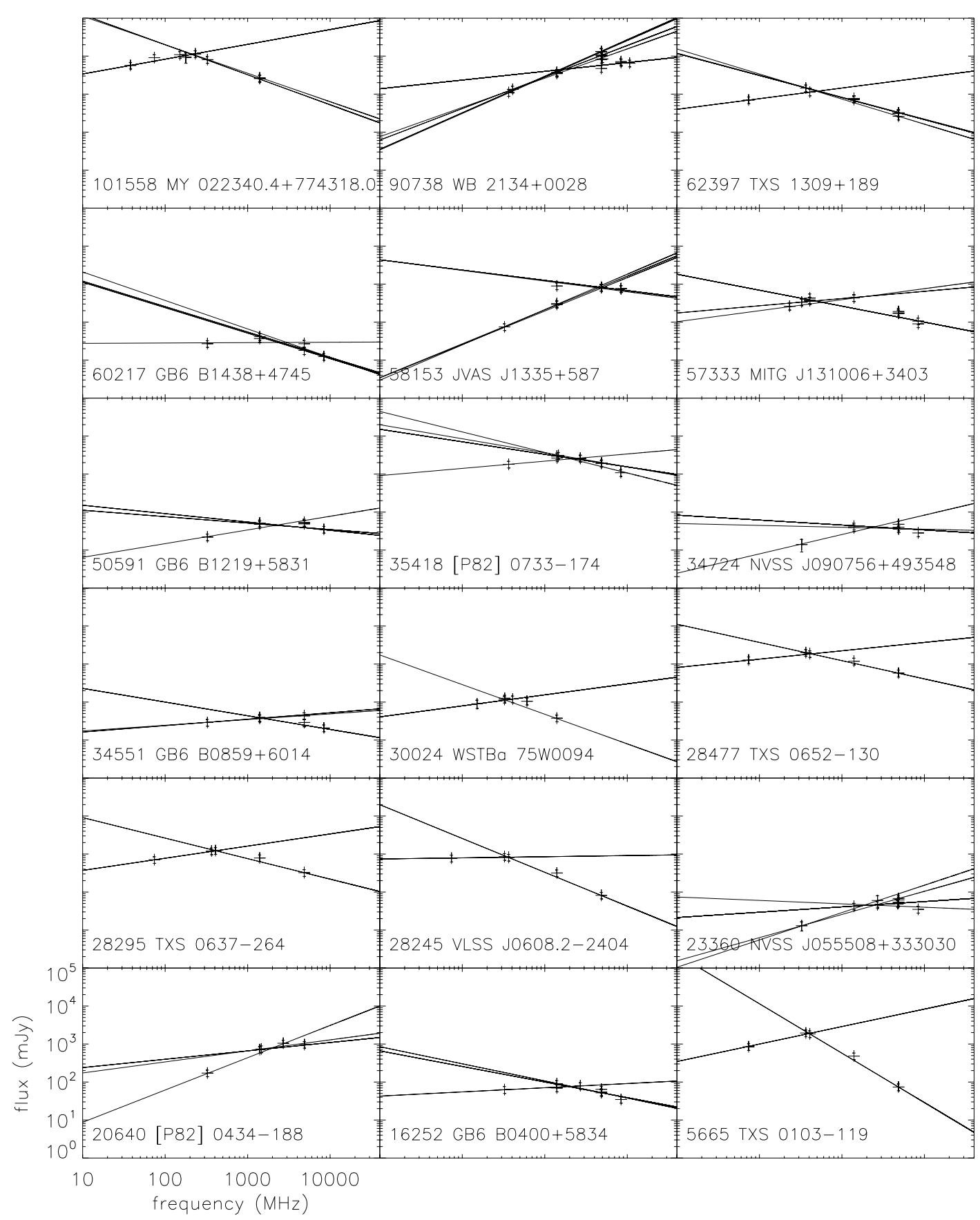

Fig. 10. Radio objects with spectral breaks identified by SPECFIND. The different lines correspond to fits of different parts of the radio spectrum. The SPECFIND V2.0 sequence number from VizieR and the name of one of the radio sources are marked in each box.

With a modest increase in the number of input sources $(\sim 8 \%)$ we could increase the number of cross-identified objects by $\sim 60 \%$. We decided not to remove extended and complex sources from SPECFIND V2.0 and therefore caution the user against a blind use of the catalogue. Typical source geometries and subsequent radio spectra are given in Sect. 4. Most frequently, the user can separate the underlying objects with the help of the SPECFIND radio spectrum and the NVSS image. Due to the larger frequency coverage of the SPECFIND entry catalogues with respect to that of the previous release spectral breaks are present in the SPECFIND V2.0 catalogue.
Acknowledgements. The new tools described in the article have been developed in the frame of the VO-TECH Design Study of the Sixth Framework Programme (Specific Support Action No 011892 "VO-TECH The European Virtual Observatory VO Technology Centre"). B.V. would like to thank T. Krichbaum and W. Reich for their valuable comments on this article.

\section{References}

Altenhoff, W. J., Downes, D., Pauls, T., et al. 1979, A\&AS, 35, 23 Altschuler, D. R. 1986, A\&AS, 65, 267

Baldwin, J. E., Boysen, R. C., Hales, S. E. G., et al. 1985, MNRAS, 217, 717 
Becker, R. H., White, R. L., \& Edwards, A. L. 1991, ApJS, 75, 1

Becker, R. H., White, R. L., Helfand, D. J., et al. 1994, ApJS, 91, 347

Benn, C. R. 1995, MNRAS, 272, 699

Benn, C. R., \& Kenderdine, S. 1991, MNRAS, 251, 253

Benn, C. R., Grueff, G., Vigotti, M., et al. 1982, 200, 747

Bennett, A. S. 1962, MNRAS, 68, 163

Bennett, C. L., Lawrence, C. R., Burke, B. F., Hewitt, J. N., \& Mahoney, J. 1986, ApJS, 61, 1

Bondi, M., Ciliegi, P., Zamorani, G., et al. 2003, A\&A, 403, 857

Bonnarel, F., Fernique, P., Bienaymé, O., et al. 2000, A\&AS, 143, 33

Browne, I. W. A., Patnaik, A. R., Wilkinson, P. N., et al. 1998, MNRAS, 293 257

Ciliegi, P., McMahon, R. G., Miley, G., et al. 1999, MNRAS, 302, 222

Cohen, A. S., Rottgering, H. J. A., Jarvis, M. J., Kassim, N. E., \& Lazio, T. J. W. 2004, ApJS, 150, 417

Cohen, A. S., Lane, W. M., Cotton, W. D., et al. 2007, AJ, 134, 1245

Coleman, P. H., Condon, J. J., \& Hazard, C. 1985, AJ, 90, 1437

Colla, G., Fanti, C., Fanti, R., et al. 1970, A\&AS, 1, 281

Colla, G., Fanti, C., Fanti, R., et al. 1972, A\&AS, 7, 1

Colla, G., Fanti, C., Fanti, R., et al. 1973, A\&AS, 11, 291

Condon, J. J., Anderson, E., \& Broderick, J. J. 1995, AJ, 109, 2318

Condon, J. J., Cotton, W. D., Greisen, E. W., et al. 1998, AJ, 115, 1693

de Breuck, C., Tang, Y., de Bruyn, A. G., Rottgering, H., \& van Breugel, W. 2002, A\&A, 394, 59

Derrière, S., Gray, N., McDowell, J. C., et al. 2004, in Astronomical Data Analysis Software and Systems (ADASS) XIII, Proceedings of the conference held 12-15 October, 2003 in Strasbourg, France, ed. Francois Ochsenbein, M. G. Allen, \& D. Egret. ASP Conf. Proc., 314 (San Francisco: ASP), 315

Douglas, J. N., Bash, F. N., Bozyan, F. A., Torrence, G. W., \& Wolfe, C. 1996, AJ, 111, 1945

Dressel, L. L., \& Condon, J. J. 1978, ApJS, 36, 53

Durdin, J. M., Pleticha, D., Condon, J. J., et al. 1975, National Astronomy and Ionosphere Center Report No. 45

Edge, D. O., Shakeshaft, J. R., McAdam, W. B., et al. 1959, MRAS, 68, 37

Fanti, C., Fanti, R., Ficarra, A., et al. 1974, A\&AS, 18, 147

Fanti, C., Mantovani, F., \& Tomasi, P. 1981, A\&AS, 43, 1

Fanti, C., Pozzi, F., Dallacasa, D., et al. 2001, A\&A, 369, 380

Ficarra, A., Grueff, G., \& Tomassetti, G. 1985, A\&AS, 59, 255

Fich, M. 1986, AJ, 92, 787

Filipovic, M. D., Haynes, R. F., White, G. L., et al. 1995, A\&AS, 111, 311

Filipovic, M. D., Bohlsen, T., Reid, W., et al. 2002, MNRAS, 335, 1085

Forkert, T., \& Altschuler, D. R. 1987, A\&AS, 70, 77

Fürst, E., Reich, W., Reich, P., et al. 1990, A\&AS, 85, 805

Garn, T., Green, D. A., Hales, S. E. G., Riley, J. M., \& Alexander, P. 2007, MNRAS, 376, 1251

Gower, J. F. R., Scott, P. F., \& Wills, D. MRAS, 1967, 71, 49

Green, D. A., \& Riley, J. M. 1995, MNRAS, 274, 324

Gregorini, L., de Ruiter, H. R., Parma, P., et al. 1994, A\&AS, 106, 1

Gregorini, L., Vigotti, M., Mack, K.-H., Zonnchen, J., \& Klein, U. 1998, A\&AS, 133,129

Gregory, P. C., \& Condon, J. J. 1991, ApJS, 75, 1011

Gregory, P. C., Scott, W. K., Douglas, K., et al. 1996, ApJS, 103, 427

Griffith, M., Langston, G., Heflin, M., et al. 1990, ApJS, 74, 129

Griffith, M., Langston, G., Heflin, M., Conner S., \& Burke B. 1991, ApJS, 75, 801

Griffith, M. R., Wright, A. E., Burke, B. F., et al. 1994, ApJS, 90, 179

Griffith, M. R., Wright, A. E., Burke, B. F., et al. 1995, ApJS, 97, 347

Hales, S. E. G., Baldwin, J. E., \& Warner, P. J. 1988, MNRAS, 234, 919

Hales, S. E. G., Masson, C. R., Warner, P. J., et al. 1990, MNRAS, 246, 256

Hales, S. E. G., Mayer, C. J., Warner, P. J., et al. 1991, MNRAS, 251, 46

Hales, S. E. G., Masson, C. R., Warner, P. J., Baldwin, J. E., \& Green, D. A. 1993, MNRAS, 262, 1057

Hales, S. E. G., Waldram, E. M., Rees, N., et al. 1995, MNRAS, 274, 447

Hales, S. E. G., Riley, J. M., Waldram, E. M., Warner, P. J., Baldwin, J. E. 2007, MNRAS, 382, 1639

Haynes, R. F., Caswell, J. L., \& Simons, L. W. J. 1979, AuJPA, 48, 1

Hopkins, A. M., Mobasher, B., Cram, L., et al. 1998, MNRAS, 296, 839

Jackson, N., Roland, J., Bremer, M., Rhee, G., \& Webb, J. 1999, A\&AS, 143, 401

Jones, P. A., \& McAdam, W. B. 1992, 80, 137
Kassim, N. E. 1988, ApJS, 68, 715

Kerton, C. R., Murphy, J., \& Patterson, J. 2007, MNRAS, 379, 289

Kollgaard, R. I., Brinkmann, W., McMath C. M., et al. 1994, ApJS, 93, 145

Kulkarni, V. K., Mantovani, F., \& Pauliny-Toth, I. I. K. 1990, A\&AS, 2, 41

Landecker, T. L., \& Caswell, J. L. 1983, AJ, 88, 1810

Langston, G. I., Heflin, M. B., Conner, S. R., et al. 1990, ApJS, 72, 621

Langston, G., Minter, A., D’Addario, L., et al. 2000, AJ, 119, 2801

Large, M. I., Cram, L. E., \& Brugess, A. M. 1991, The Observatory, 111, 72

Laurent-Muehleisen, S. A., Kollgaard, R. I., Ryan, P. J., et al. 1997, A\&AS, 122, 235

Lawrence, C. R., Bennett, C. L., Garcia-Barreto, J. A., Greenfield, P. E., \& Burke, B. F. 1983, ApJS, 51, 67

Leahy, D. A., \& Roger, R. S. 1996, A\&AS, 115, 345

Ledlow, M. J., \& Owen, F. N. 1995, AJ, 109, 853

Louys, M., Richards, A., Bonnarel, F., et al. 2008, Data Model for Astronomical DataSet Characterisation, Version 1.13, IVOA Documents, http://www . ivoa.net/Documents/latest/CharacterisationDM.html

Mauch, T., Murphy, T., Buttery, H. J., et al. 2003, MNRAS, 342, 1117

Morganti, R., Garrett, M. A., Chapman, S., et al. 2004, A\&A, 424, 371

Murphy, T., Mauch, T., Green, A., et al. 2007, MNRAS, 382, 382

Myers, S. T., Jackson, N. J., Browne, I. W. A., et al. 2003, MNRAS, 341, 1

Niklas, S., Klein, U., Braine, J., et al. 1995, A\&AS, 114, 21

Ochsenbein, F., Bauer, P., \& Marcout, J. 2000, A\&AS, 143, 23

O'Dea, C. P. 1998, PASP, 110, 493

Oort, M. J. A. 1987, A\&AS, 71, 221

Oort, M. J. A., Steemers, W. J. G., \& Windhorst, R. A. 1988, A\&AS, 73, 103

Otrupcek, R., \& Wright, A. E. 1991, PASAu, 9, 1700

Owen, F. N., White, R. A., Hilldrup, K. C., et al. 1982, AJ, 87, 1083

Paladini, R., Burigana, C., Davies, R. D., et al. 2003, A\&A, 397, 213

Patnaik, A. R., Browne, I. W. A., Wilkinson, P. N., et al. 1992, MNRAS, 254, 655

Pearson, T. J. 1975, MNRAS, 171, 475

Pearson, T. J. 1978, MNRAS, 182, 273

Perley, R. A. 1982, AJ, 87, 859

Pilkington, J. D. H., \& Scott, P. F. 1965, MRAS, 69, 183

Quiniento, Z. M., \& Cersosimo, J. C. 1993, A\&AS, 97, 435

Reich, W., Furst, E., Steffen, P., Reif, K., \& Haslam, C. G. T. 1984, A\&AS, 58, 197

Reich, W., Reich, P., \& Furst, E. 1990, A\&AS, 83, 539

Reich, P., Reich, W., \& Fuerst, E. 1997, A\&AS, 126, 413

Reich, W., Fuerst, E., Reich, P., et al. 2000, A\&A, 363, 141

Rengelink, R. B., Tang, Y., de Bruyn, A. G., et al. 1997, A\&AS, 124, 259

Righetti, G., Giovannini, G., \& Feretti, L. 1988, A\&AS, 74, 315

Roettgering, H. J. A., Lacy, M., Miley, G. K., Chambers, K. C., \& Saunders, R. 1994, A\&AS, 108, 79

Slee, O. B. 1995, AuJPh, 48, 143

Slee, O. B., Perley, R. A., \& Siegman, B. C. 1989, AuJPh, 42, 633

Tasse, C., Cohen, A. S., Roettgering, H. J. A., et al. 2006, A\&A, 456, 791

Tasse, C., Roettgering, H. J. A., Best, P. N., et al. 2007, A\&A, 471, 1105

Taylor, J. H., Manchester, R. N., \& Lyne, A. G. 1993, ApJS, 88, 529

Taylor, A. R., Goss, W. M., Coleman, P. H., van Leeuwen, J., \& Wallace, B. J. 1996, ApJS, 107, 239

Vollmer, B., Davoust, E., Dubois, P., et al. 2005a, A\&A, 431, 1177

Vollmer, B., Davoust, E., Dubois, P., et al. 2005b, A\&A, 436, 757

Vollmer, B., Krichbaum, T. P., Angelakis, E., et al. 2008, A\&A, 489, 49

Waldram, E. M., Pooley, G. G., Grainge, K. J. B., et al. 2003, MNRAS, 342, 915

Walterbos, R. A. M., Brinks, E., \& Shane, W. W. 1985, A\&AS, 61, 451

Wieringa, M. H. 1993, Bull. Inf. CDS 43, 17

Wieringa, M. H., \& Ekers, R. D. 2000, A\&AS, 146, 41

White, R. L., \& Becker, R. H. 1992, ApJS, 79, 331

White, R. L., Becker, R. H., Helfand, D. J., et al. 1998, ApJ, 475, 47

White, R. L., Becker, R. H., \& Helfand, D. J. 2005, AJ, 130, 586

Wilkinson, P. N., Browne, I. W. A., Patnaik, A. R., Wrobel, J. M., \& Sorathia, B. 1998, MNRAS, 300, 790

Windhorst, R. A., van Heerde, G. M., Katgert, P. 1984, A\&AS, 58, 1

Wright, A. E., Griffith, M. R., Burke, B. F., et al. 1994, ApJS, 91, 11

Wright, A. E., Griffith, M. R., Hunt, A. J., et al. 1996, ApJS, 103, 145

Zhang, X., Zheng, Y., Chen, H., et al. 1997, A\&AS, 121, 59

Zhang, X., Reich, W., Reich, P., et al. 2003, A\&A, 404, 57

Zoonematkermani, S., Helfand, D. J., Becker, R. H., White, R. L., \& Perley, R. A. 1990, ApJS, 74, 181 